\title{
The influence of illumination upon the temporal patterning of responses in squirrel and owl monkeys'
}

\author{
WALTER ISAAC, ${ }^{2}$ Emory University, Atlanta, Ga. 30322
}

The temporal patterning of responses within a fixed-interval reinforcement situation was studied in the nocturnal Owl monkey and the diumal Squirrel monkey. The response rates of both groups were found to vary, but in opposite directions, with illumination conditions, but no evidence of temporal patterning was observed in the Owl monkeys.

Illumination conditions have been shown to influence the temporal patterning of responses in a fixed interval operant conditioning situation by Isaac \& Kendall (1967). They found that the total response frequency in the rat was higher in the dark than in the light. In addition, the acceleration in response frequency over the interreinforcement interval was greater in the dark. If their interpretation of their data is appropriate it should be possible to generalize their findings to other animals who show greatest activity in the dark and an opposite effect of illumination should be seen in an animal whose greatest activity is in the light.

In an attempt to evaluate such a notion two members of the Cebidae family, the nocturnal Aotes (Owl monkey) and the diurnal Saimiri (Squirrel monkey), were employed as Ss in a situation similar to that employed by Isaac and Kendall.

\section{SUBJECTS}

Three members of each genus served as Ss. All were young adult males. Because of marked circadian variations the nocturnal Aotes were trained between 6:00 PM and midnight while the Saimiri were trained between 6:00 AM and noon.

\section{PROCEDURE}

The Ss were studied in an enclosure into which their carrying cage was inserted. The interior of the enclosure was painted flat white. The doors of the carrying cage were modified so that a plastic lever $4 \times 1$ in. extended into the interior at one end $2 \frac{1}{2} \mathrm{in}$. above the floor; a reinforcer through which a small quantity of sweetened fruit juice could be offered extended $2 \mathrm{in}$. up from the floor at the other end. The larger enclosure was 16 in. square and 22 in. high. The carrying cage measured $15 \times 9 \times 9$ in. Illumination of $70 \mathrm{ft}-\mathrm{c}$ could be provided by a light source located $7 \mathrm{ft}$ above the carry ing cage.

All Ss were studied for a $30-\min$ period daily. Conditions of light and dark were presented on alternate days. During the final phase of the study a single reinforcement was available during each 80-sec interval. The number of responses made in each 20-sec quarter of this interval were recorded for each experimental session. The reinforcement was available during the first quarter.

After training under conditions of light and dark in excess of two months the performance of the Ss had stabilized and the data to be reported was gathered. Data from the last four experimental sessions under each illumination condition were evaluated by analysis of variance.

\section{RESULTS}

As apparent in Fig. 1, analysis of variance showed the main effects of genus $(\mathrm{F}=25.72 ; \mathrm{df}=1,4)$, illumination $(\mathrm{F}=51.16$; $\mathrm{df}=1,124)$, and $20-\mathrm{sec}$ quarters of the $80-\mathrm{sec}$ interval $(\mathrm{F}=10.75 ; \mathrm{df}=3,24)$ to exert effects significant at less than the $1 \%$ level. The difference between replications was not significant. The interactions between levels of illumination and genus $(F=217.96 ; d f=1,124)$, intervals and genus $(F=6.52$; $\mathrm{df}=3,124)$, and level of illumination and intervals $(F=11.96$; $\mathrm{df}=3,124)$ were significant at less than the $1 \%$ level. The interaction between genus and illumination and intervals $(F=4.09, d f=3,124)$ was significant at less than the $1 \%$ level.
The magnitude of the effects of the levels of illumination used was not consistent, as evidenced by an interaction between illumination levels and replications $(F=3.78 ; \mathrm{df}=3,124)$ significant at the $5 \%$ level; however, the relative effects of illumination did not vary in a consistent fashion, as evidenced by an interaction between levels of illumination, replications, and genus $(\mathrm{F}=6.27 ; \mathrm{df}=3,124)$ that was significant at less than the $1 \%$ level.

\section{DISCUSSION}

Contrary to the subjective impression of the Owl monkey as a sluggish, slow-moving $S$ that may be formed from observing his activity during the normal daylight hours, the Owl monkey demonstrated a high response rate when compared to the Squirrel monkey in the present situation. A marked circadian rhythm (Worthington, 1968) makes it necessary to observe these Ss at night to properly evaluate their capacities.

The expectation that the presence or absence of illumination would influence in an opposite fashion the rate of responding of the two monkeys in a fixed-interval operant situation was realized. In the nocturnal Owl monkey the highest response rate was observed in the dark, as was the case with the rat (Isaac \& Kendall, 1967). The Squirrel monkey showed. the highest response rate in the light. An additional differential effect of illumination upon the two groups of Ss is apparent in their respective low response rate conditions. Under darkness, the Squirrel monkeys almost eliminated their responses, while under light, the Owl monkeys merely reduced their response rate. Thus, the effects of illumination in the nocturnal and diumal Ss differed in direction but were not mirror images.

A further difference of interest was the total lack of temporal patterning of responses observed in the Owl monkeys. The Squirrel monkeys showed the typical change in response rate within the interreinforcement period in the light. The stability of this phenomenon is demonstrated by the lack

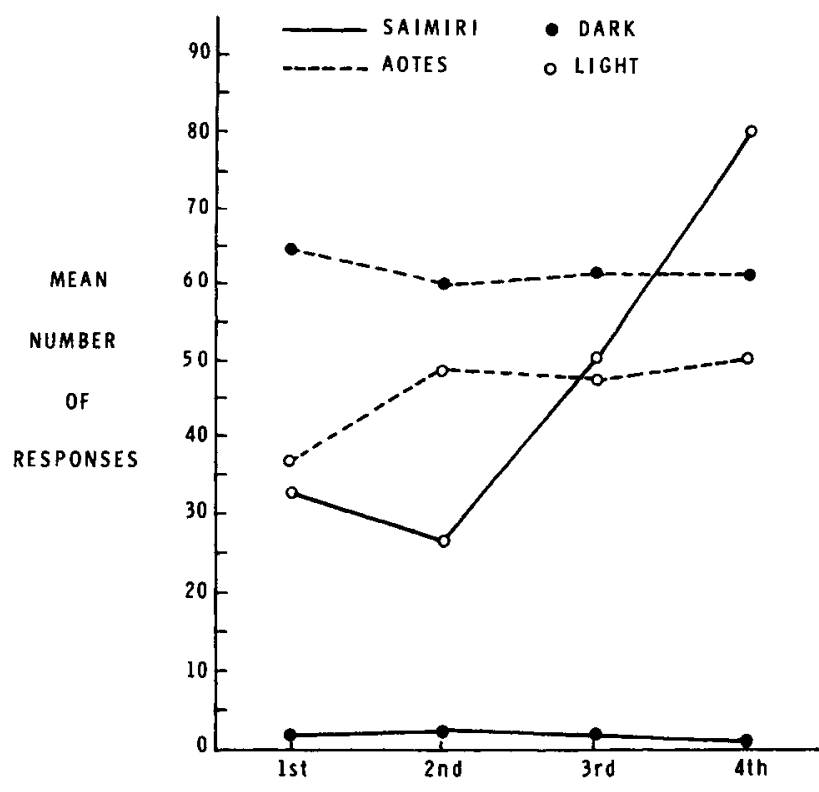

QUARTERS OF AN 80 SEC, FIXED INTERVAL

Fig. 1. Distribution of responses within the interreinforcement interval as a function of illumination. 
of change in this phenomenon over the four replications analyzed as well as those earlier in training.

\section{REFERENCES}

ISAAC, W., \& KENDALL, N. P. Sensory stimulation and timing beh avior. Psychonomic Science, 1967, 8, 41-42.
WORTHINGTON, C. S. Paradoxical illumination effects upon the behavior of two species of Cebid monkeys. Unpublished dissertation, Emory University, 1968.

\section{NOTES}

1. This work was supported by Research Grant MH 04539 from the National Institute of Mental Health.

2. Present address: University of Georgia, Athens Ga. 30601

(Continued from page 242)

Rate stabilization, habituation, recovery, and extinction sessions were all of 15-min duration. CS presentations of 1-min occurred in the eighth and twelfth minute of the habituation and extinction sessions. The rate stabilization and recovery sessions contained no CS presentations.

In Session d, Groups CT-T and CT-L received a 43-min, 20-sec CER training session consisting of 20 tone-shock pairings with a variable intertrial interval (VITI) of $2 \mathrm{~min}$. The tone CS duration was $10 \mathrm{sec}$, and the US, presented in the final second of the $\mathrm{CS}$, was a $2-\mathrm{mA}, 1-\mathrm{sec}$ shock. The two pseudoconditioning groups (PCT-T and PCT-L) were treated similarly to the CT-T and CT-L groups except that the tone and shock occurred randomly with respect to each other. Conditioning and pseudoconditioning occurred with the dipper inaccessible to Ss.

In Session e, 10 fear-conditioning trials were given to all Ss during a 22-min, 40-sec session with the dipper down. Trials were separated by a VITI of $2-\mathrm{min}$ and consisted of a $15-\mathrm{sec}$ change in light intensity coterminating with a 1-mA shock of 1 -sec duration. Ss were left in the experimental chambers for $15 \mathrm{~min}$ after the end of the session; no events were programmed during this period.

During testing, Ss received seven 10 -sec tones with a VITI of 2-min in a 15-min, 10-sec period in which the dipper was inaccessible. A 15-min licking session followed in which a 60-sec CS occurred following the seventh and eleventh minute. The CS was the tone for the CT-T and PCT-T Ss and the light for the CT-L and PCT-L Ss.

The data were analyzed in the form of suppression ratios, $D /(B+D)$, where $D$ represents the number of responses during the CS and $B$ the number of responses in an equal period of time immediately preceding it.

\section{RESULTS}

Data from the groups tested with the tone suggested that the free tones given prior to testing did induce emotionality (see Fig. 1, Panel B). Both the CT-T and the PCT-T groups suppressed more to the first $\mathrm{CS}$ in testing than to the last $C S$ in tone-habituation $(\mathrm{F}=44.10, \quad \mathrm{df}=1 / 22, \quad \mathrm{p}<.001, \quad$ and $\mathrm{F}=24.3, \mathrm{df}=1 / 22, \mathrm{p}<.001$ for CT-T and PCT-T, respectively). But surprisingly, these two groups did not differ in suppression to the first tone in testing $(F<1.0)$. Because the PCT-T and CT-T groups feared the tone equally, the free tones should not have differentially affected suppression to the light in testing for the CT-L and PCT-L groups. This prediction is confirmed by the nonsignificant difference $(F<1.0)$ between these groups on suppression to the first light CS following the free tones (see Panel A).

Since the free tones induced emotionality in both the CT-L and PCT-L groups, the motivational hypothesis requires that these groups show more suppression to the first CS presentation in testing than to the last presentation of the same CS in the extinction session. Both the CT-L and the PCT-L groups licked more during the first CS in testing than in the last $C S$ in extinction $(F=8.83, \mathrm{df}=1 / 44, p=.005$, and
$\mathrm{F}=21.8, \mathrm{df}=1 / 44, \mathrm{p}<.00 \mathrm{l}$, respectively), contradicting the motivational interpretation of CER enhancement.

All groups suppressed to the light in the extinction session and showed a marked decrement in suppression over extinction trials. Both of these effects were highly reliable. Finally, Ss receiving the conditioning procedure with the tone did not suppress more to the light in extinction than Ss receiving the pseudoconditioning procedure with the tone $(F=1.0)$; that is, no transfer was observed. However, in view of the fact that the CT-T and PCT-T groups could not be distinguished later on the basis of their suppression to the tone, this result is difficult to interpret.

\section{DISCUSSION}

The major finding of this research was that emotionality produced by a tone CS did not enhance suppression to another CS as was expected on the basis of the emotionality hypothesis. Because of the many procedural differences between Hoffman's free-shock study and the present one, it cannot be determined whether the discrepant results are due to the method of inducing emotionality or to some other factor. In this regard, Quinsey \& Ayres (1969), using a paradigm similar to that used here, were unable to enhance a partially extinguished CER with free shock but succeeded in arresting CER extinction, thereby enhancing the CER relative to that of a spontaneous recovery control. We tentatively conclude, therfore, that fear induction is not sufficient to enhance a partially extinguished CER or to stop its further extinction.

It was observed earlier that in Hoffman's experiment both a motivational and an associative interpretation lead to the same prediction. This is not the case in the present study because the tone-shock pairings occurred a full day before the light-shock pairings. It is unlikely that the tones were part of the stimulus context in which the CER to the light was learned. In the present study, the associative hypothesis predicts, therefore, that the free-tone presentations should not have enhanced suppression to the light.

The apparent pseudoconditioning of the PCT-T Ss may be due to remote associations facilitated by the relatively large number of high intensity shocks they received. Quinsey \& Ayres (1969), using a similar procedure but with fewer shocks of lower intensity, failed to demonstrate pseudoconditioning.

\section{REFERENCES}

HOFFMAN, H. S. The stimulus generalization of conditioned suppression. In D. 1. Mostofsky (Ed.), Stimulus generalization. Stanford: Stanford University Press, 1965. Pp. 356-372.

QUINSEY, V. L., \& AYRES, J. J. B. Shock-induced facilitation of a partially extinguished CER. Psychonomic Science, 1969, 14, 213-214. NOTE

1. This study represents part of Vernon L. Quinsey's M.S. thesis. It was done under the direction of John J. B. Ayres and was partially supported by a grant to John J. B. Ayres from the Faculty Research Council of the University of Massachusetts. 